\title{
IncRNA MIR4435-2HG promotes the progression of liver cancer by upregulating B3GNT5 expression
}

\author{
YUNGANG ZHU $^{1 *}$, BAOGUO LI ${ }^{2 *}$, GUOPING XU ${ }^{3}$, CHANGRUI HAN $^{1}$ and GANG XING ${ }^{1}$ \\ ${ }^{1}$ Radiology Department, Tianjin Teda Hospital, Tianjin 300457; ${ }^{2}$ Department of Interventional Treatment, \\ National Clinical Research Center for Cancer, Key Laboratory of Cancer Prevention and Therapy, \\ Tianjin's Clinical Research Center for Cancer, Tianjin Medical University Cancer Institute and Hospital, Tianjin 300060; \\ ${ }^{3}$ Medical Imaging Department, Second Hospital of Tianjin Medical University, Tianjin 300211, P.R. China
}

Received April 6, 2021; Accepted October 4, 2021

DOI: $10.3892 / \mathrm{mmr} .2021 .12554$

\begin{abstract}
Several studies have indicated that dysregulation of long non-coding RNAs (lncRNAs) participates in the initiation and progression of cancer. The lncRNA MIR4435-2HG was previously reported to act as an oncogene in human cancer, including liver cancer. However, its role in the pathogenesis in liver cancer is largely unclear. The present study aimed to reveal the molecular mechanism by which MIR4435-2HG regulates liver cancer. The expression levels of MIR4435-2HG in liver cancer and adjacent normal tissues were analyzed using The Cancer Genome Atlas database. MIR4435-2HG expression was validated by reverse transcription-quantitative polymerase chain reaction (RT-qPCR) in cancer cells in vitro. The target genes of MIR4435-2HG were predicted using bioinformatics analysis. Interactions between miR-136-5p, MIR4435-2HG and B3GNT5 were detected using luciferase reporter assays, and their effects on cell viability, migration and invasion were assessed using Cell Counting Kit-8, wound healing and Transwell assays. The effects of miR-136-5p and MIR4435-2HG on B3GNT5 expression were confirmed by western blot analysis. The results revealed that MIR4435-2HG expression was upregulated in primary liver cancer and liver cancer cell lines, and was positively associated with advanced tumor stage, metastasis and poor prognosis in patients with liver cancer. Knockdown of MIR4435-2HG significantly inhibited the proliferation, migration and invasion of liver cancer cells. Furthermore, miR-136-5p was determined to be a direct target of MIR4435-2HG and suppressed MIR4435-2HG expression by binding with the seed region of the 3 '-UTR
\end{abstract}

Correspondence to: Professor Gang Xing, Radiology Department, Tianjin Teda Hospital, 65 Third Avenue, Binhai, Tianjin 300457, P.R. China

E-mail: doctorxing2021@126.com

${ }^{*}$ Contributed equally

Key words: liver cancer, MIR4435-2HG, microRNA-136-5p, B3GNT5, proliferation of MIR4435-2HG in liver cancer cells. Functional studies showed that the inhibitory effects of MIR4435-2HG knockdown on cell proliferation, migration and invasion were significantly rescued by inhibiting miR-136-5p. Furthermore, the target gene, B3GNT5, of miR-136-5p was confirmed by bioinformatics analysis and RT-qPCR. In addition, B3GNT5 expression was regulated by the MIR4435-2HG/miR-136-5p axis. In conclusion, the present study indicated that MIR4435-2HG facilitated the progression of liver cancer via the MIR4435-2HG/miR-136-5p/B3GNT5 axis, which demonstrated that MIR4435-2HG may be a potential biomarker for the prognosis and treatment of liver cancer.

\section{Introduction}

Liver cancer is a serious public health problem worldwide and is the fourth leading cause of cancer-related deaths worldwide (1). Among the different subtypes of liver cancer, hepatocellular carcinoma (HCC) is the main cause of primary liver cancer (2). HCC accounts for $\sim 80 \%$ of all liver cancer cases (3). Despite the improved therapeutic strategies for patients with liver cancer, the 5-year survival rates are far from the expected result due to the high rates of recurrence and metastasis of liver cancer (4). Thus, it is important to explore the underlying molecular mechanisms of liver cancer to improve the survival rate of patients.

Long non-coding RNAs (lncRNAs) are a type of RNA. Although they do not encode proteins, lncRNAs still play significant roles in gene expression and cellular biological processes $(5,6)$. In previous years, it has been reported that lncRNAs are involved in the progression of human diseases (7). For example, in lymph node metastasis of bladder cancer, lncRNA ELNAT1 has been shown to promote UBC9 expression to catalyze SUMOylation, lymphangiogenesis and lymph node metastasis of breast cancer (8). IncRNA lnc030 has also been reported to be highly expressed in breast cancer stem cells in vitro and in vivo, and to cooperate with poly(rC)-binding protein 2 to stabilize squalene epoxidase mRNA (9). Furthermore, lncRNA CRNDE may bind to the splicing protein SRSF6 to inhibit its function and affect alternative splicing by mediating the autophagic flux in chemoresistant gastric cancer (GC) cells (10). IncRNA MIR4435-2HG has been found to be 
highly expressed and directly involved in the tumorigenesis of several types of cancer, including gastric cancer, non-small cell lung cancer and colorectal cancer (11-13). Notably, the progression and metastasis of GC have been shown to be regulated by the lncRNA MIR4435-2HG via the Wnt/ $\beta$-catenin signaling pathway (12). Knockdown of MIR4435-2HG may also suppress colorectal cancer cell proliferation, invasion and migration via the miR-206/YAP1 axis (11). In addition, MIR4435-2HG expression has been reported to be increased in liver cancer $(14,15)$. However, the functions of MIR4435-2HG in liver cancer require further investigation.

The present study aimed to detect the expression levels of MIR4435-2HG in liver cancer and to explore the molecular mechanism underlying the regulatory effect of MIR4435-2HG on the progress of liver cancer.

\section{Materials and methods}

Clinical specimens. Liver cancer tissues and adjacent normal tissues were collected from patients with liver cancer $(n=40)$ at the Second Hospital of Tianjin Medical University (Tianjin, China) and were stored in liquid nitrogen until further analysis. Tissue samples were collected between January 2017 and December 2019. Of the 40 patients, 18 were women and 22 were men; 17 patients were $<50$ years old and 23 patients were $\geq 50$ years old; the mean age of all patients was 54 years old. None of the patients received radiotherapy or chemotherapy before surgical resection. The inclusion criteria were as follows: i) Patients diagnosed with primary liver cancer; and ii) knew the purpose of the research and provided written informed consent. The exclusion criteria were as follows: i) Patients diagnosed with other tumors or liver insufficiency; ii) patients had mental illness; and iii) patients also had other types of cancer. The present study was approved by the Ethics Committee of the Second Hospital of Tianjin Medical University (approval no. KY2020K093). All patients provided written informed consent prior to the study.

Cell culture and transfection. The normal liver cell line THLE-2, and human liver cancer cell lines HCCLM3 and HepG2 were purchased from the American Type Culture Collection. The cells were cultured in RPMI-1640 medium (Gibco; Thermo Fisher Scientific, Inc.) supplemented with $10 \%$ fetal bovine serum (FBS; Gibco; Thermo Fisher Scientific, Inc.), $100 \mathrm{U} / \mathrm{ml}$ penicillin and $100 \mu \mathrm{g} / \mathrm{ml}$ streptomycin in an incubator at $37^{\circ} \mathrm{C}$ with $5 \% \mathrm{CO}_{2}$. All cell lines were authenticated using short tandem repeat profiling.

For cell transfection assays, scrambled shRNA negative control (shNC), two shMIR4435-2HG (sh2HG-1 and sh2HG-2), empty pcDNA vector (pcDNA3), pcDNA MIR4435-2HG (MIR4435-2HG), pcDNA B3GNT5 (B3GNT5), miR-NC mimics, miR-NC inhibitor, miR-136-5p mimics and miR-136-5p inhibitor were constructed and purchased from Shanghai GeneChem Co., Ltd. The sequences were as follows: miR-NC mimics, 5'-UUCUCCGAACGUGUCACGUTT-3'; miR-136-5p mimics, 5'-ACUCCAUUUGUUUUGAUGAUG GA-3'; miR-NC inhibitor, 5'-CAGUACUUUUGUGUAGUA CAA-3'; miR-136-5p inhibitor, UCCAUCAUCAAAACAAAU GGAGU-3'. HCCLM3 and HepG2 cells at 70\% confluence were transfected with $1 \mu \mathrm{g}$ shRNA and plasmids, or miRNA mimics/inhibitor at $37^{\circ} \mathrm{C}$ using Lipofectamine ${ }^{\circledR} 3000$ reagent (Invitrogen; Thermo Fisher Scientific, Inc.). After $48 \mathrm{~h}$, the cells were harvested for other assays.

Reverse transcription-quantitative polymerase chain reaction $(R T-q P C R)$. Total RNA, including miRNA, was extracted from tissues and cells using TRIzol ${ }^{\circledR}$ reagent (Invitrogen; Thermo Fisher Scientific, Inc.). cDNA was then synthesized from mRNA using the MMLV Reverse kit (Promega Corporation) according to the manufacturer's instructions. RT reagents used for miRNA (cat. no. D1801) were provided by HaiGene Co., Ltd and RT was performed according to manufacturer's protocol. Gene expression was detected by qPCR using SYBR ${ }^{\circledR}$ Premix $\mathrm{EX} \mathrm{Taq}^{\mathrm{TM}}$ kit (Takara Bio, Inc.). The thermocycling conditions were as follows: i) $95^{\circ} \mathrm{C}$ for $5 \mathrm{~min}$; ii) 40 cycles at $95^{\circ} \mathrm{C}$ for $10 \mathrm{sec}, 60^{\circ} \mathrm{C}$ for $30 \mathrm{sec}$ and $72^{\circ} \mathrm{C}$ for $30 \mathrm{sec}$. The expression levels of MIR4435-2HG and B3GNT5 were normalized to those of GAPDH, whereas U6 served as the internal control for miR-136-5p. The relative expression levels of the genes were calculated using the $2^{-\Delta \Delta \mathrm{Cq}}$ method (16). The primer sequences used in the present study are as follows: MIR4435-2HG, forward, 5'-CTGGATGGTCGCTGCTTTTTA-3' and reverse 5'-AGGGGGATGAGTCGTGATTT-3'; miR-136-5p, forward 5'-ACTCCATTTGTTTTGATGATGGA-3' and reverse 5'-TGG TGTCGTGGAGTCG-3'; B3GNT5, forward 5'-GGGCCTCGC TACCAATACTTG-3' and reverse 5'-CGGAACGTCGATCAT AGTTTTCA-3'; GAPDH, forward 5'-AATGGACAACTG GTCGTGGAC-3' and reverse 5'-CCCTCCAGGGGATCT GTTTG-3'; and U6, forward 5'-AGTAAGCCCTTGCTGTCA GTG-3' and reverse 5'-CCTGGGTCTGATAATGCTGGG.

Western blotting. Proteins were extracted using radioimmunoprecipitation assay buffer (Beyotime Institute of Biotechnology). Protein concentrations were quantified using a BCA Protein Assay Kit (Beyotime Institute of Biotechnology). Subsequently, the proteins $(30 \mu \mathrm{g})$ were separated by sodium dodecyl sulfate-polyacrylamide gel electrophoresis on $10 \%$ gels and transferred to a polyvinylidene difluoride membrane. The membranes were blocked with 5\% non-fat milk for $1 \mathrm{~h}$ at room temperature and incubated with the corresponding primary antibodies: Anti-B3GNT5 (1:1,000; cat. no. PA5-26653; Thermo Fisher Scientific, Inc.) and anti-GAPDH (1:5,000; cat. no. ab8245; Abcam) at $4^{\circ} \mathrm{C}$ overnight. After washing the membrane with Tris-buffered saline with $0.1 \%$ Tween-20, the membranes were incubated with the corresponding horseradish peroxidase-conjugated secondary antibodies: Goat anti-rabbit secondary antibody (1:3,000; cat. no. 7074; Cell Signaling Technology, Inc.) and horse anti-mouse (1:3,000; cat. no. 7076; Cell Signaling Technology, Inc.) at room temperature for $1 \mathrm{~h}$. Protein bands were imaged using an Enhanced Chemiluminescence Kit (Beyotime Institute of Biotechnology). The intensity of the protein bands was analyzed using ImageJ 1.51 software (National Institutes of Health).

Cell Counting Kit-8 (CCK-8) assay. The CCK-8 kit (Dojindo Molecular Technologies, Inc.) was used to measure the prolferation ability of transfected liver cancer cells. Transfected cells $\left(5 \times 10^{3} /\right.$ well) were seeded into 96 -well plates and incubated for the indicated time periods $(24,48$ and $72 \mathrm{~h})$ at 
$37^{\circ} \mathrm{C}$. After incubation, $10 \mu 1 \mathrm{CCK}-8$ solution was added to the corresponding wells for $2 \mathrm{~h}$ at $37^{\circ} \mathrm{C}$. The optical density values at $450 \mathrm{~nm}$ were measured using a microplate reader. Cell proliferation ability was calculated according to the manufacturer's instructions.

Wound healing assay. HCCLM3 and HepG2 cells were seeded in six-well plates. The wound was made when the cells reached $>90 \%$ confluence and the scratched cells were cultured in serum-free medium. The width of the scratch was imaged at 0 and $24 \mathrm{~h}$ using an inverted light microscope. The relative mobility of the cells was then calculated using ImageJ 1.51 software.

Transwell assay. Transwell assays were conducted to assess cell migration and invasion. For the migration assay, the upper chambers of the Transwell inserts were not coated with Matrigel, whereas in the invasion assay the upper chambers were coated with Matrigel at $37^{\circ} \mathrm{C}$ for $7 \mathrm{~h}$. HCCLM3 and HepG2 cells $\left(1 \times 10^{5}\right)$ were added into the upper chambers of Transwell inserts in $200 \mu \mathrm{l}$ medium. The bottom chambers of the Transwell inserts were filled with $600 \mu \mathrm{l}$ medium supplemented with $10 \%$ FBS. After $48 \mathrm{~h}$, the cells in the upper chambers were fixed with $4 \%$ paraformaldehyde for $10 \mathrm{~min}$ at room temperature and stained with $0.4 \%$ crystal violet for $10 \mathrm{~min}$ at room temperature. Images of the migrated or invaded cells were captured and cells were counted in five random fields using an inverted light microscope.

Dual-luciferase reporter assay. The binding sites between genes were identified using bioinformatics software starBase3.0 (http://starbase.sysu.edu.cn). The wild-type (WT) or mutant (MUT) 3'-UTRs of MIR4435-2HG or B3GNT5 were cloned into the pGL3-luciferase vector (Promega Corporation). Briefly, to synthesize MUT sequences, the nucleotide sequences of MIR4435-2HG or B3GNT5, which exhibit complementary binding sequences to miR-136-5p, were changed by replacing the original base sequences so they were no longer complementary to miR-136-5p. Subsequently, miR-NC, miR-136-5p mimic, WT-MIR4435-2HG, MUT-MIR4435-2HG, WT-B3GNT5 or MUT-B3GNT5 were co-transfected into the indicated groups of 293T cells (American Type Culture Collection) with the indicated groups using Lipofectamine 3000. The groups were as follows: WT-MIR4435-2HG + miR-NC mimics, WT-MIR4435-2HG + miR-136-5p mimics, MUT-MIR4435-2HG + miR-NC mimics, MUT-MIR4435-2HG + miR-136-5p mimics, WT-B3GNT5 + miR-NC mimics, WT-B3GNT5 + miR-136-5p mimics, MUT-B3GNT5 + miR-NC mimics and MUT-B3GNT5 + miR-136-5p mimics). After $48 \mathrm{~h}$, luciferase activity was measured using a dual-luciferase reporter assay system (Promega Corporation) according to the manufacturer's instructions. Luciferase activity was normalized to Renilla luciferase activity.

Bioinformatics analysis. MIR4435-2HG expression in The Cancer Genome Atlas (TCGA) was analyzed using Gene Set Cancer Analysis (GSCA; http://bioinfo.life.hust.edu. $\mathrm{cn} / \mathrm{GSCA} / \# /)$ in all cancer datasets, Gene Expression Profiling Interactive Analysis 2 (GEPIA2; http://gepia.cancer-pku.cn/) in the liver hepatocellular carcinoma (LIHC) dataset and DeepBase 3.0 (http://rna.sysu.edu.cn/deepbase3/index.html) in the LIHC dataset. The relationship between MIR4435-2HG expression and cancer metastasis was studied using the Human Cancer Metastasis Database (http://hcmdb.i-sanger. com/index). The relationship between MIR4435-2HG expression and prognosis of patients with liver cancer was analyzed using GEPIA2. Potential target miRNAs of MIR4435-2HG were predicted using the starBase 3.0 website (http://starbase. sysu.edu.cn). Target genes of miR-136-5p were predicted by starBase 3.0, miRWalk 3.0 (http://mirwalk.umm.uniheidelberg.de/), TargetScan 7.1 (http://www.targetscan. org/vert_71/), miRDB 6.0 (http://mirdb.org/) and ONCOMIR (http://www.oncomir.org/oncomir/index.html) databases. The expression of B3GNT5 and its effects on the prognosis of patients with liver cancer were analyzed using the GEPIA2 database. Heat map analysis of the expression of targets of miR-136-5p was performed using the UALCAN database (http://ualcan.path.uab.edu/). The variation in copy number, including single nucleotide variation (SNV) and copy number variation (CNV), and methylation of targets of miR-136-5p were analyzed using the GSCA database; SNV and CNV of genes were obtained by selecting different commands in the GSCA database. The correlation between MIR4435-2HG and B3GNT5 expression was determined using the GEPIA database.

Statistical analysis. Data are shown as the mean \pm standard deviation and were analyzed using SPSS 19.0 (IBM Corp.) and GraphPad Prism 8.0 (GraphPad Software, Inc.). Differences between normal and tumor tissues were analyzed using a paired t-test. Other differences between two independent groups were analyzed using an unpaired t-test. Differences between multiple groups were analyzed using one-way ANOVA followed by Tukey's post hoc test. The survival analysis was performed using the Kaplan-Meier method followed by a log-rank test in the GEPIA database. Linear regression analysis was used to detect the association between MIR4435-2HG and miR-136-5p. Pearson's correlation analysis was used to detect the correlation between MIR4435-2HG and B3GNT5 gene expression levels. Spearman's correlation analysis was used to analyze the correlation between B3GNT5 methylation and expression at the mRNA level. $\mathrm{P}<0.05$ was considered to indicate a statistically significant difference.

\section{Results}

MIR4435-2HG is upregulated in liver cancer tissue in TCGA database. First, MIR4435-2HG expression across TCGA cancer types was analyzed using the GSCA database. The results indicated that the expression levels of MIR4435-2HG were significantly upregulated in a various types of cancer, including liver cancer (Fig. 1A). TCGA data in the GEPIA database further demonstrated that the expression levels of MIR4435-2HG were increased in liver cancer tissues compared with those in adjacent normal tissues (Fig. 1B). Moreover, increased MIR4435-2HG expression was associated with liver cancer progression (Fig. 1C). Kaplan-Meier analysis showed that patients with liver cancer with higher MIR4435-2HG expression had poor overall survival (OS), 
A

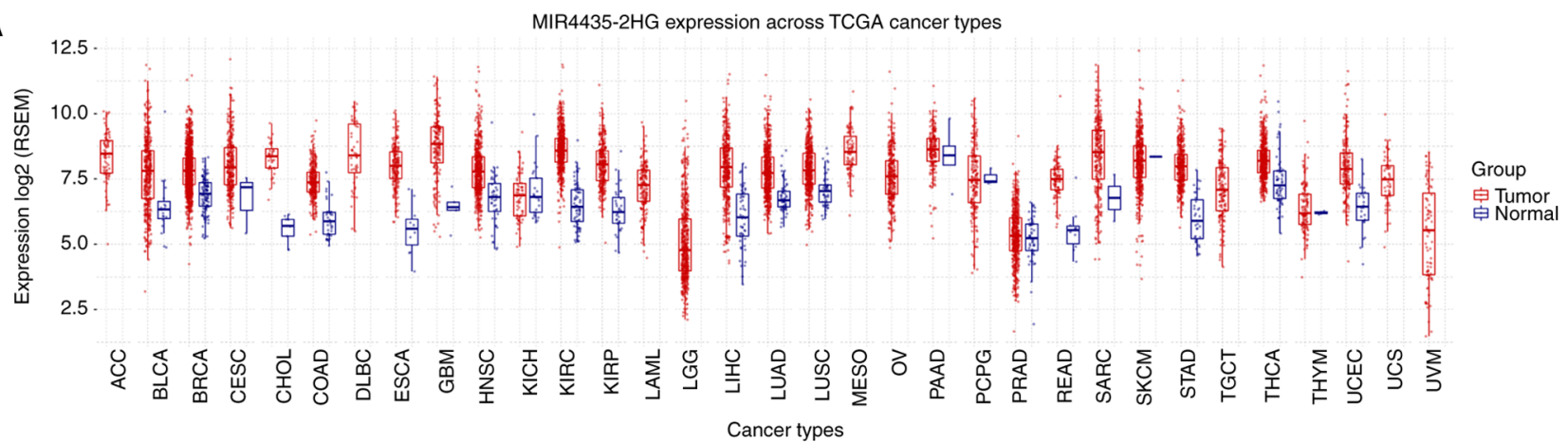

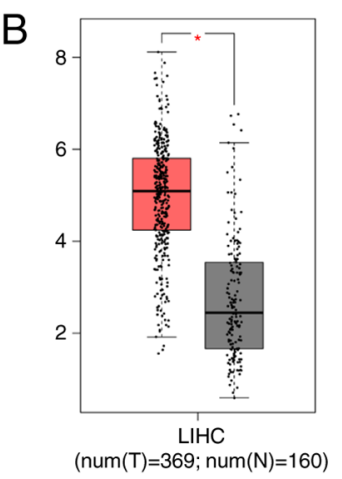

C

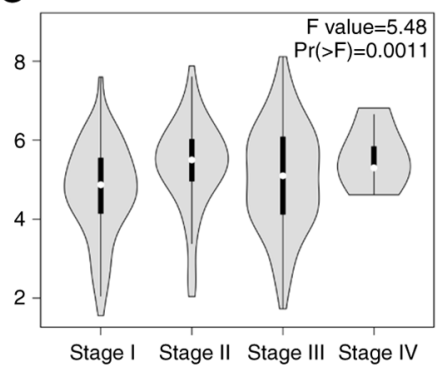

E

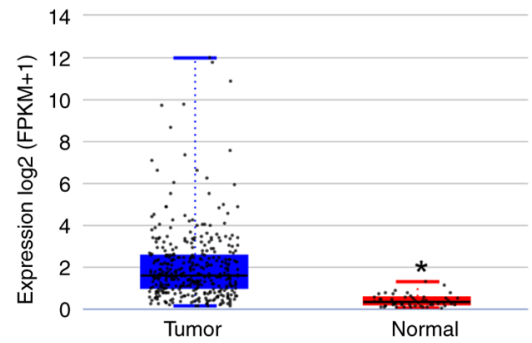

D
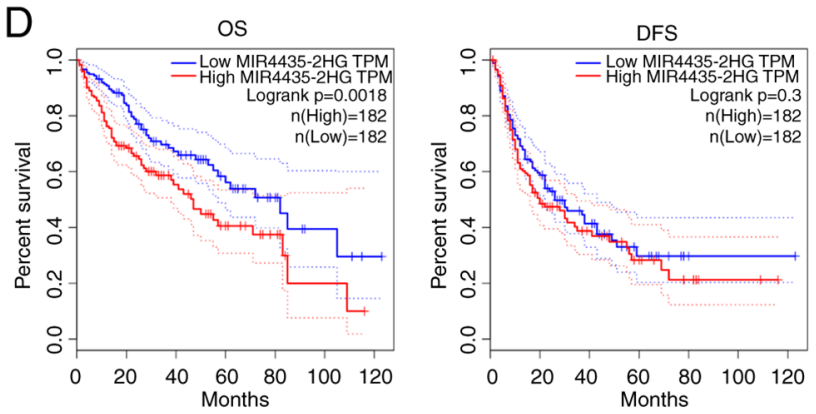

$\mathrm{F}$

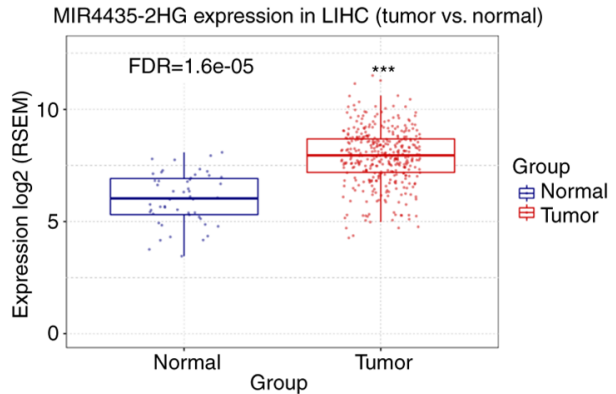

G

MIR4435-2HG expression

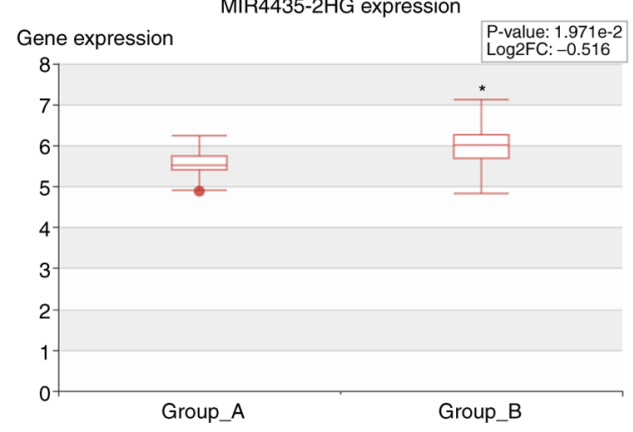

Figure 1. MIR4435-2HG expression is upregulated in liver cancer tissues. (A) Expression analysis of MIR4435-2HG across TCGA cancer types in the GSCA database. (B) Expression of MIR4435-2HG in liver cancer tissues and normal tissues was evaluated using the GEPIA database. * $<0.05$ vs. normal tissues. (C) Expression of MIR4435-2HG was analyzed in different tumor TNM stages in the GEPIA database. (D) OS and DFS of patients with liver cancer with different expression patterns of MIR4435-2HG in the GEPIA database. (E and F) MIR4435-2HG expression analysis in DeepBase and GSCA databases. ${ }^{*} \mathrm{P}<0.05,{ }^{* * *} \mathrm{P}<0.001$ vs. normal tissues. (G) MIR4435-2HG expression was associated with liver cancer metastasis in the Human Cancer Metastasis Database database. "P<0.05 vs. Group A. Group A, non-metastasis; Group B, metastasis. TCGA, The Cancer Genome Atlas; GSCA, Gene Set Cancer Analysis; GEPIA, Gene Expression Profiling Interactive Analysis; OS, overall survival; DFS, disease-free survival; LIHC, liver hepatocellular carcinoma.

but it had no obvious effect on disease-free survival (DFS) (Fig. 1D). Furthermore, MIR4435-2HG expression was found to be increased in patients with liver cancer in the DeepBase and GSCA databases, respectively (Fig. 1E and F). Of note, in patients with liver cancer, the expression of MIR4435-2HG was further increased with the occurrence of metastasis (Fig. 1G). These data suggested that MIR4435-2HG may be a pivotal oncogene in liver cancer.
MIR4435-2HG knockdown inhibits the malignant behavior of liver cancer cells. In the present study, the expression levels of MIR4435-2HG in liver cancer tissues and cell lines were measured by RT-qPCR. The results revealed that MIR4435-2HG expression levels were significantly upregulated in liver cancer tissues and cell lines compared with those in normal tissues and cell lines (Fig. 2A and B). Subsequently, the role of MIR4435-2HG was investigated in 
A

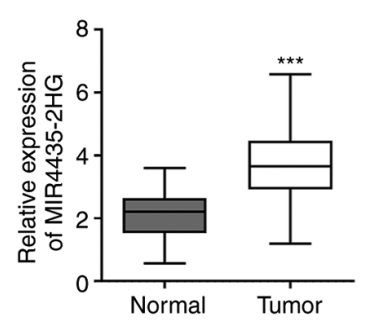

E

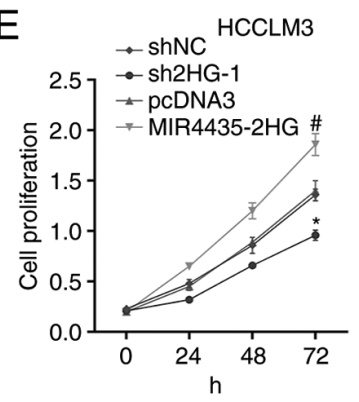

G

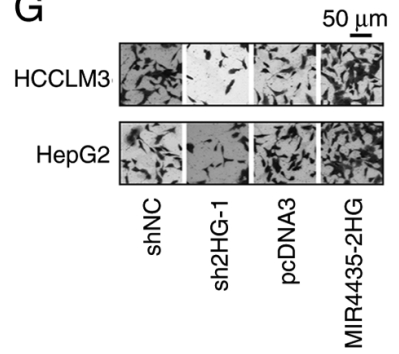

B
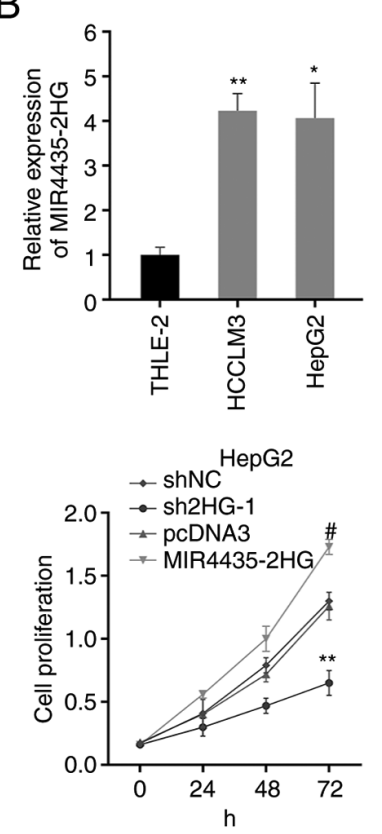

F

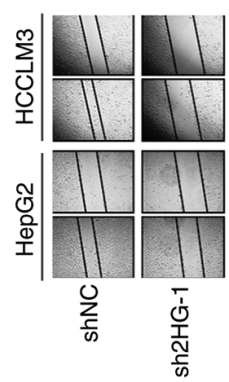

$\mathrm{H}$

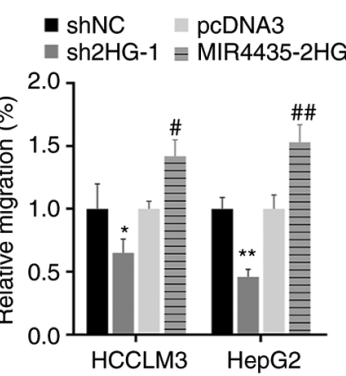

C
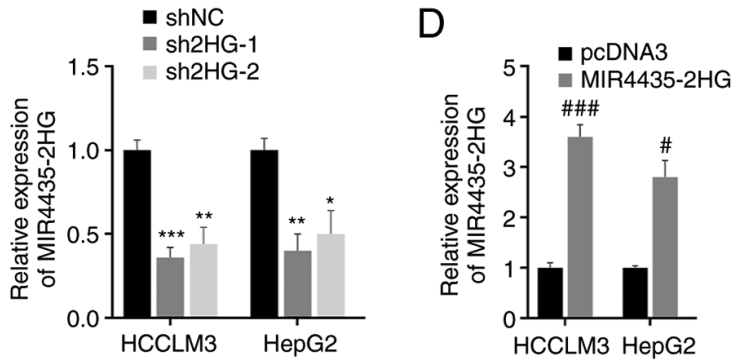

- shNC $\square$ pCDNA3

an2HG-1 $\equiv$ MIR4435-2HG
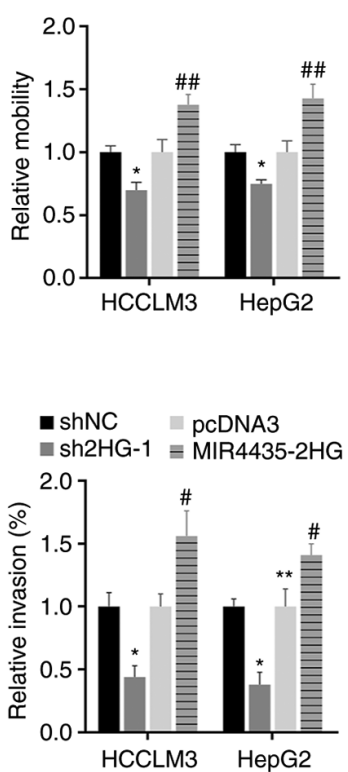

Figure 2. Knockdown of MIR4435-2HG inhibits the proliferation, migration and invasion of liver cancer cells. (A) MIR4435-2HG expression was detected in liver cancer and normal liver tissues via RT-qPCR. ${ }^{* * *} \mathrm{P}<0.001$ vs. normal tissues. (B) MIR4435-2HG expression in normal liver cells and liver cancer cell lines. ${ }^{*} \mathrm{P}<0.05,{ }^{* *} \mathrm{P}<0.01$ vs. THLE-2 cells. (C and D) After transfection with sh2HG-1, sh2HG-2, MIR4435-2HG or corresponding NCs, the expression levels of MIR4435-2HG were examined using RT-qPCR. ${ }^{*} \mathrm{P}<0.05,{ }^{* *} \mathrm{P}<0.01,{ }^{* * *} \mathrm{P}<0.001$ vs. shNC, ${ }^{\#} \mathrm{P}<0.05,{ }^{\# \# \#} \mathrm{P}<0.001$ vs. pcDNA3. (E) Proliferation was measured using the CCK-8 assay in liver cancer cells with indicated transfections. (F) Wound healing assay was used to assess cell migration (magnification, $x 50$ ). $\left(\mathrm{G}\right.$ and $\mathrm{H}$ ) The effects of MIR4435-2HG on cell migration and invasion were measured by Transwell assays (magnification, $\mathrm{x} 400$ ). ${ }^{*} \mathrm{P}<0.05,{ }^{* *} \mathrm{P}<0.01 \mathrm{vs}$. shNC, ${ }^{\#} \mathrm{P}<0.05,{ }^{\# \#} \mathrm{P}<0.01$ vs. pcDNA3. RT-qPCR, reverse transcription-quantitative PCR; sh, short hairpin RNA; sh2HG, shMIR4435-2HG; NC, negative control.

liver cancer cells. Liver cancer cells were transfected with shNC, shMIR4435-2HG (sh2HG-1 and sh2HG-2), pcDNA3 or MIR4435-2HG. It was revealed that the expression levels of MIR4435-2HG were significantly affected by sh2HG-1, sh2HG-2 and MIR4435-2HG (Fig. 2C and D). Since sh2HG-1 produced a more obvious inhibitory effect on MIR4435-2HG, sh2HG-1 was used in subsequent experiments. In addition, proliferation was measured using the CCK- 8 assay. The results revealed that sh2HG-1 resulted in decreased proliferation, whereas overexpression of MIR4435-2HG in cancer cells exerted the opposite effect (Fig. 2E). Wound healing and Transwell assays were performed to assess cell migration and invasion capacities, and revealed that sh2HG-1 reduced the migration and invasion abilities of liver cancer cells (Fig. 2F-H). However, in liver cancer cells transfected with MIR4435-2HG, migration and invasion were significantly potentiated (Fig. 2F-H). These results suggested that the silencing of MIR4435-2HG may reduce the malignant biological behavior of liver cancer cells by influencing cell proliferation, migration and invasion.
miR-136-5p is a target of MIR4435-2HG. To explore the potential molecular mechanisms of MIR4435-2HG in the regulation of liver cancer progression, its target genes were predicted using bioinformatic tools. By analyzing previous research reports (17-20), it was found that miR-136-5p (identified as a target of MIR4435-2HG using bioinformatics analysis) expression was significantly decreased in various types of cancer, indicating that miR-136-5p may act as a tumor suppressor. However, the role of miR-136-5p in the liver has rarely been studied. Therefore, miR-136-5p was chose as a potential target of MIR4435-2HG for further analysis; the potential binding site of miR-136-5p on MIR4435-2HG is shown in Fig. 3A. First, the expression levels of miR-136-5p in liver cancer tissues were detected; it was revealed that miR-136-5p expression was significantly downregulated in liver cancer tissues (Fig. 3B). To confirm the direct binding between miR-136-5p and MIR4435-2HG, 293T cells were co-transfected with miR-136-5p mimics and WT/MUT-MIR4435-2HG luciferase reporter plasmids. The luciferase reporter assay showed that miR-136-5p significantly suppressed the luciferase 

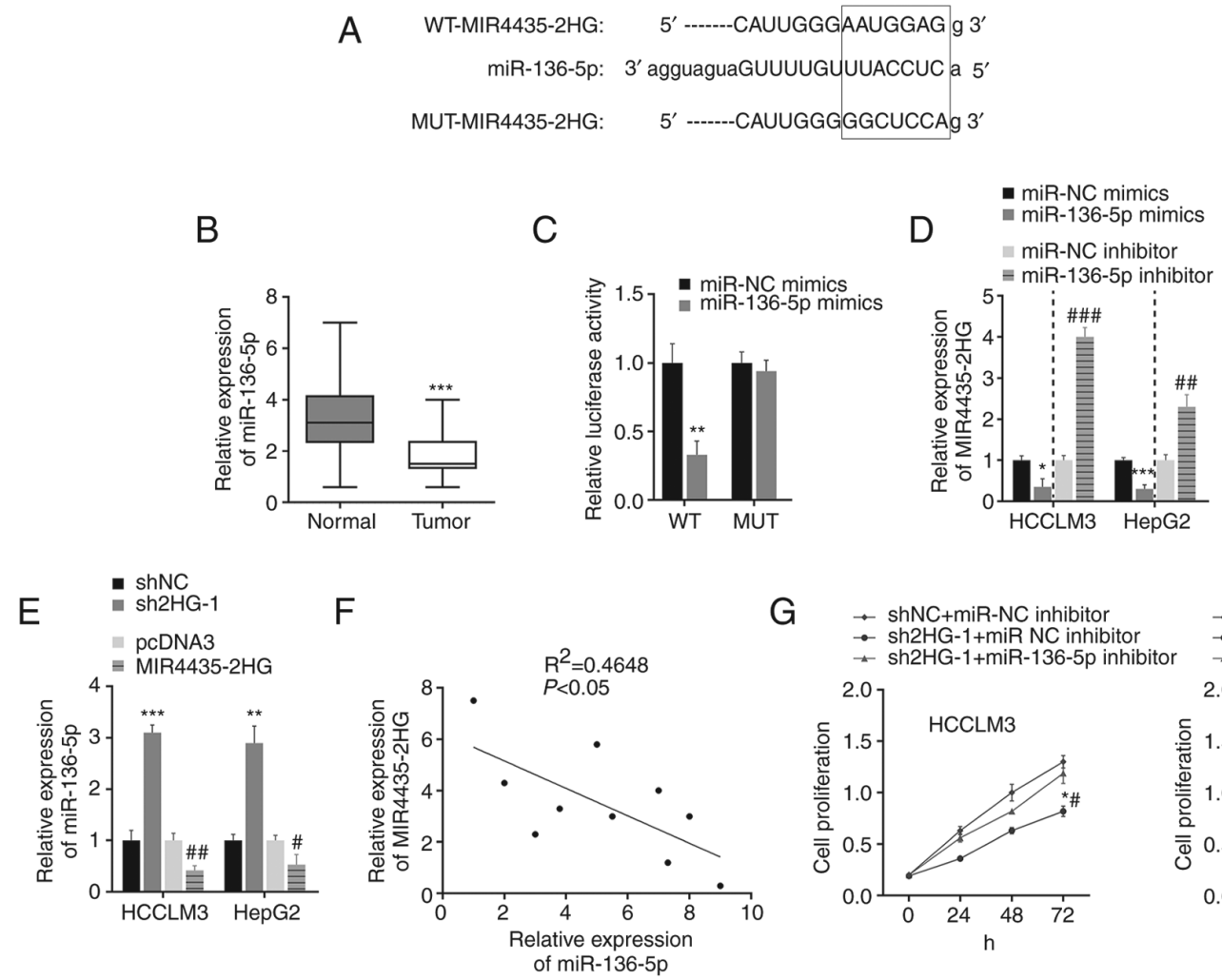

$\rightarrow$ shNC+miR-NC inhibitor
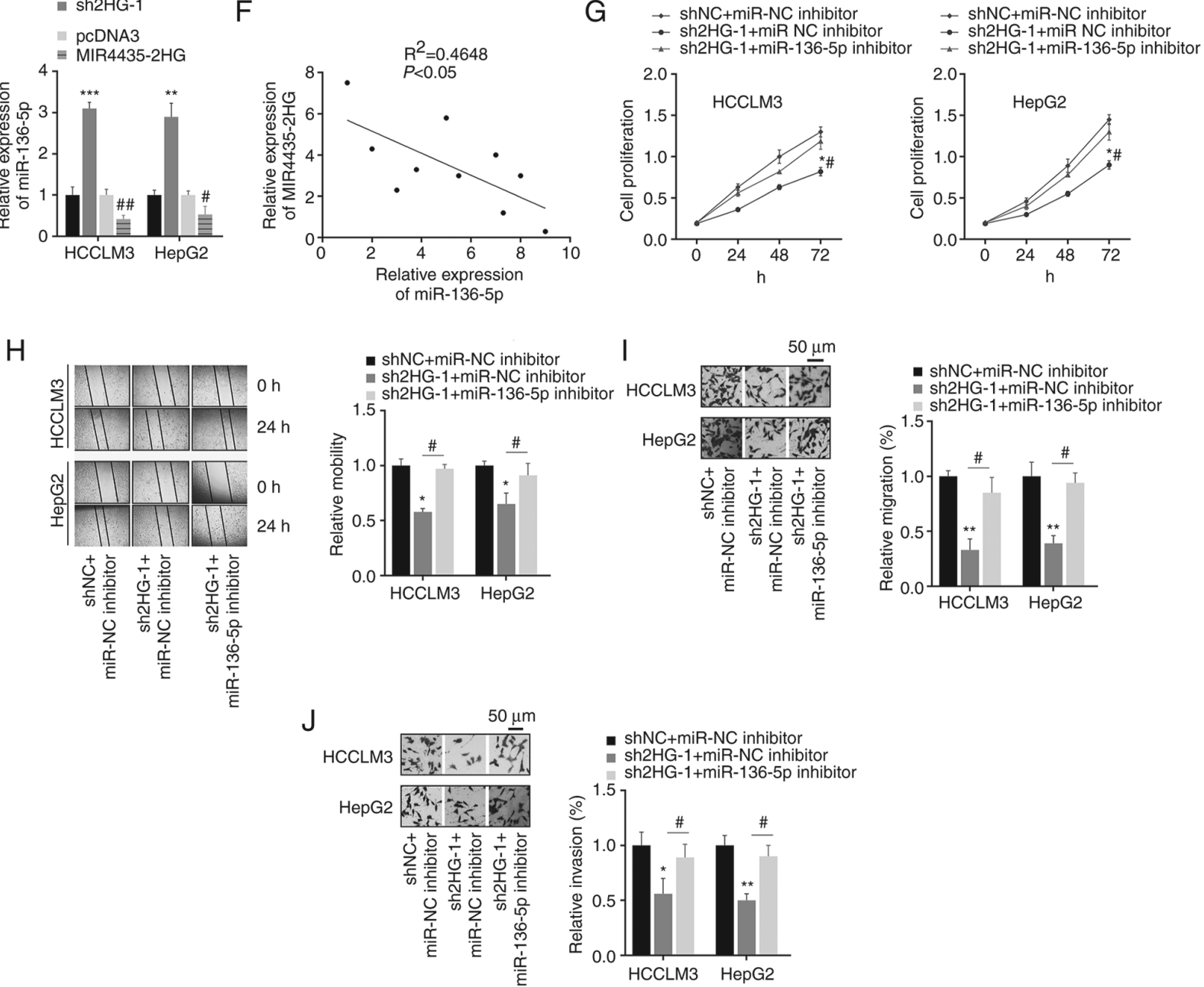

Figure 3. MIR4435-2HG targets miR-136-5p. (A) The WT or MUT binding sites of MIR4435-2HG on miR-136-5p. (B) miR-136-5p expression in liver cancer and normal tissues. ${ }^{* * *} \mathrm{P}<0.001$ vs. normal tissues. (C) Dual-luciferase reporter assay indicated that the luciferase activities were decreased after co-transfection of WT-MIR4435-2HG and miR-136-5p mimics. ** P $<0.01$ vs. miRNA NC mimics. (D) The effect of miR-136-5p on MIR4435-2HG expression. " $\mathrm{P}<0.05$, ${ }^{* * *} \mathrm{P}<0.001$ vs. miRNA NC mimics; ${ }^{\# \#} \mathrm{P}<0.05,{ }^{\# \# \#} \mathrm{P}<0.001$ vs. miRNA NC inhibitor. (E) MIR4435-2HG regulated miR-136-5p expression in liver cancer cells. ${ }^{* *} \mathrm{P}<0.01,{ }^{* * *} \mathrm{P}<0.001$ vs. shNC; ${ }^{\#} \mathrm{P}<0.05,{ }^{\# \#} \mathrm{P}<0.01$ vs. pcDNA3. (F) Negative association between MIR4435-2HG and miR-136-5p expression in liver cancer tissues. (G) Transfection with sh2HG inhibited cell proliferation, which was rescued by miR-136-5p inhibitor. (H-J) Transfection with sh2HG affected cell migration and invasion by targeting miR-136-5p. Magnification, $x 50 .{ }^{*} \mathrm{P}<0.05,{ }^{* *} \mathrm{P}<0.01$ vs. shNC + miR-NC inhibitor; ${ }^{*} \mathrm{P}<0.05$ vs. sh2HG-1 + miR-136-5p inhibitor. miR/miRNA, microRNA; WT, wild-type; MUT, mutant; NC, negative control; sh, short hairpin RNA sh2HG, shMIR4435-2HG.

activity of cells transfected with WT-MIR4435-2HG, but not MUT-MIR4435-2HG (Fig. 3C). These findings indicated that MIR4435-2HG can target and sponge miR-136-5p. Cancer cells were transfected with miR-136-5p mimics or inhibitor before functional analysis of miR-136-5p (Fig. S1A and B). It was found that transfection of cells with miR-136-5p mimics significantly reduced the expression of MIR4435-2HG, whereas transfection with a miR-136-5p inhibitor significantly 
A

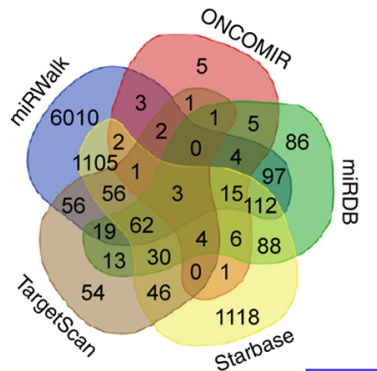

WT-B3GNT5: 5' uaCAUAGAGGAAUAUAAUAAGGAG a 3 miR-136-5p: 3' agGUA-GUAGUUUUGU--JUACCUC a 5' WT-B3GNT5: 5' UaCAUAGAGGAAUAUAAUCGCAAGA a $3^{\prime \prime}$

B

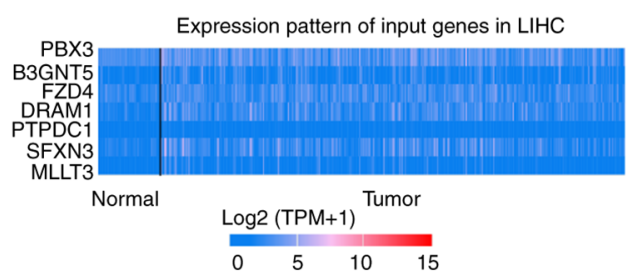

C

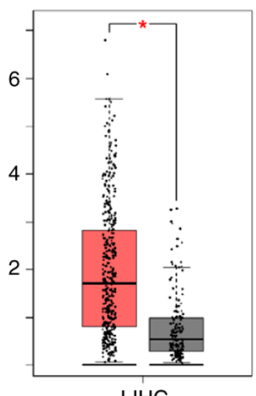

(num $(T)=369 ; \operatorname{num}(\mathrm{N})=160)$
D
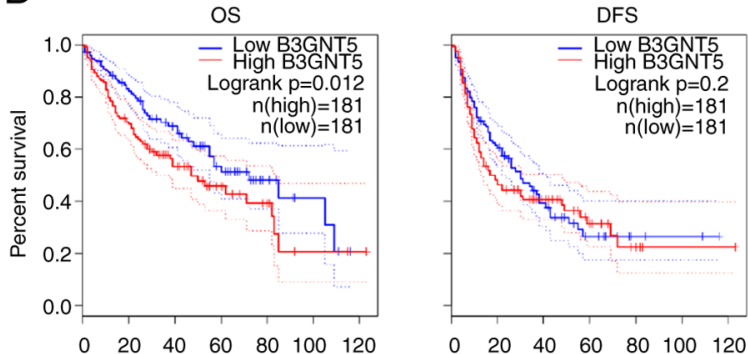

$\begin{array}{lllllll}0 & 20 & 40 & 60 & 80 & 100 & 120\end{array}$
E

\begin{tabular}{|c|c|c|}
\hline \multirow[b]{2}{*}{ PTPDC1 } & \multicolumn{2}{|c|}{ SNV percentage heatmap } \\
\hline & 2 & ○ \\
\hline MLLT3 & 2 & ப் 10 \\
\hline FZD4 & 2 & 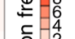 \\
\hline B3GNT5 & 1 & 을 $\mathbb{1}_{0}^{2}$ \\
\hline SFXN3 & 0 & $\sum^{2}$ \\
\hline PBX3 & 0 & \\
\hline
\end{tabular}

F

B3GNT5: (Somatic mutation rate: $0.27 \%$ )

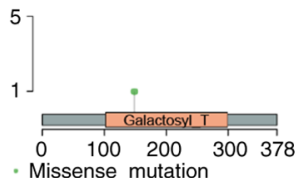

G

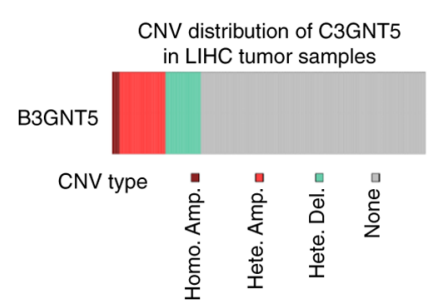

I

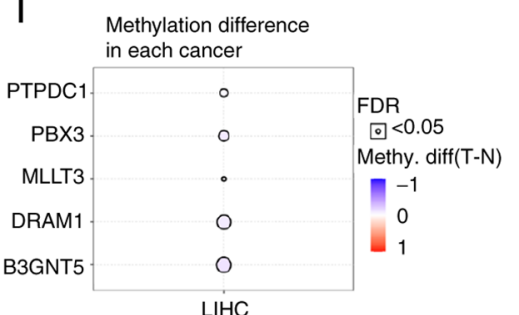

H $\quad 1.00$

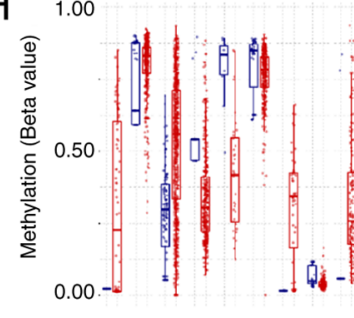

B3GNT5 methylation across TCGA caner types

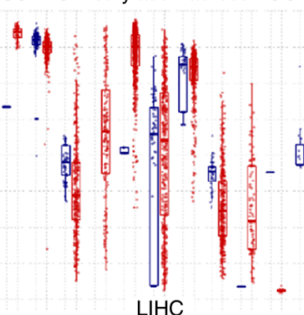

$J$

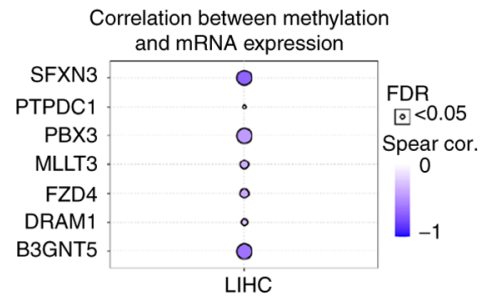

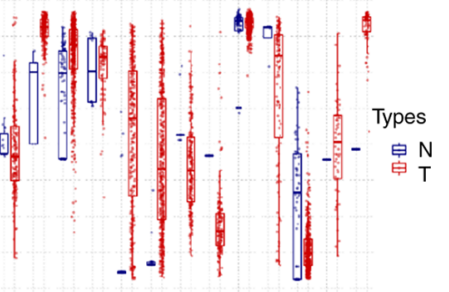

$\mathrm{K}$
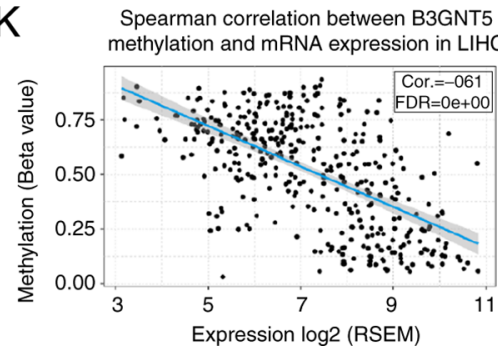

Figure 4. Prediction and analysis of potential target genes of miR-136-5p. (A) Target genes of miR-136-5p were predicted using bioinformatics tools (starBase, miRWalk, TargetScan, ONCOMIR and miRDB). (B) Expression profile of seven target genes of miR-136-5p was analyzed using the UALCAN database. (C) Increased B3GNT5 expression was detected in LIHC in the GEPIA database. (D) OS and DFS of patients with liver cancer with different expression profiles of B3GNT5. GSCA database was used to study the SNV, copy number alteration and methylation levels of target genes. (E) SNV percentage of target genes in liver cancer. (F) Missense mutation of B3GNT5 in liver cancer. (G) CNV types of B3GNT5 in liver cancer. (H) B3GNT5 methylation levels across TCGA cancer types. (I) Methylation difference of target genes in liver cancer. (J) Correlation between methylation and mRNA expression levels of target genes of miR-136-5p. (K) Negative correlation between B3GNT5 methylation level and mRNA expression. "P<0.05. miR, microRNA; GEPIA, Gene Expression Profiling Interactive Analysis; OS, overall survival; DFS, disease-free survival; SNV, single nucleotide variation; CNV, copy number variation; TCGA, The Cancer Genome Atlas; WT, wild-type; MUT, mutant; FDR, false discovery rate; LIHC, liver hepatocellular carcinoma.

upregulated MIR4435-2HG expression (Fig. 3D). Similarly, the expression of miR-136-5p in liver cancer cells was affected by the overexpression of MIR4435-2HG (Fig. 3E); this may be due to the fact that MIR4435-2HG can competitively bind to miR-136-5p, which leads to the downregulation of miR-136-5p. Furthermore, linear regression analysis validated the moderate negative association between MIR4435-2HG and miR-136-5p expression in liver cancer tissues (Fig. 3F). Functionally, the inhibition of cell proliferation, migration and invasion via MIR4435-2HG knockdown were partially restored by the miR-136-5p inhibitor (Fig. 3G-J). In general, these data indicated that MIR4435-2HG affected the proliferation, migration and invasion of liver cancer cells by negatively regulating miR-136-5p expression.

Expression analysis of the potential miR-136-5p target B3GNT5 in liver cancer. Next, the molecular mechanism of miR-136-5p in liver cancer was further revealed by predicting its downstream targets using bioinformatics tools (starBase, miRWalk, TargetScan, ONCOMIR and miRDB; Fig. 4A). Among these targets, gene sets that existed in different databases and had no more than 10 target genes were selected 
A

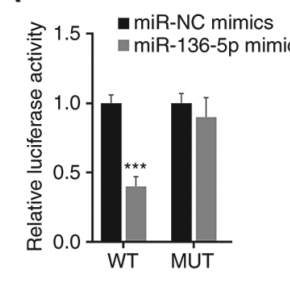

F

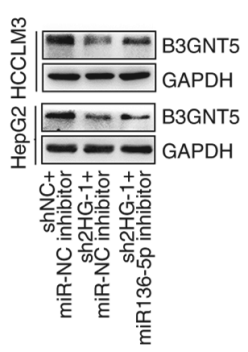

B

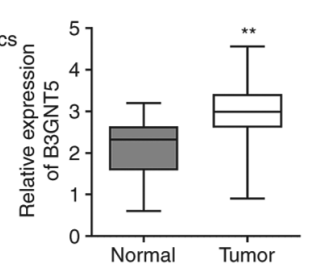

C
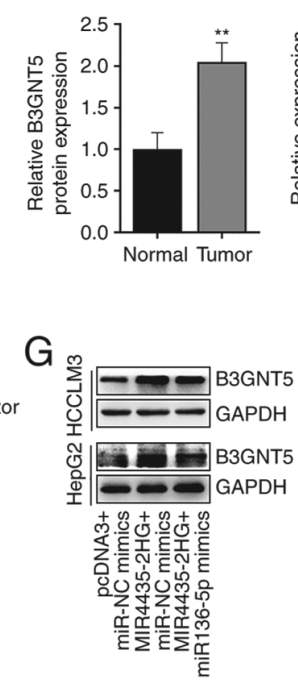

D

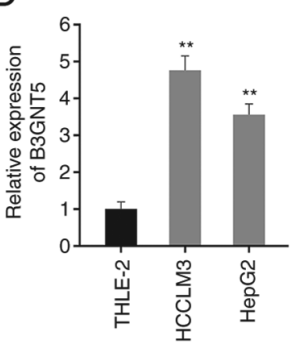

E

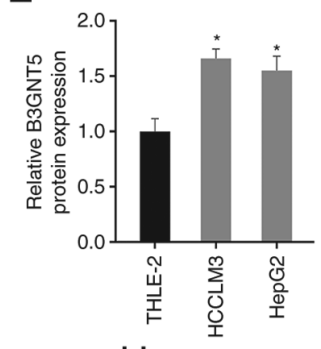

$\mathrm{H}$
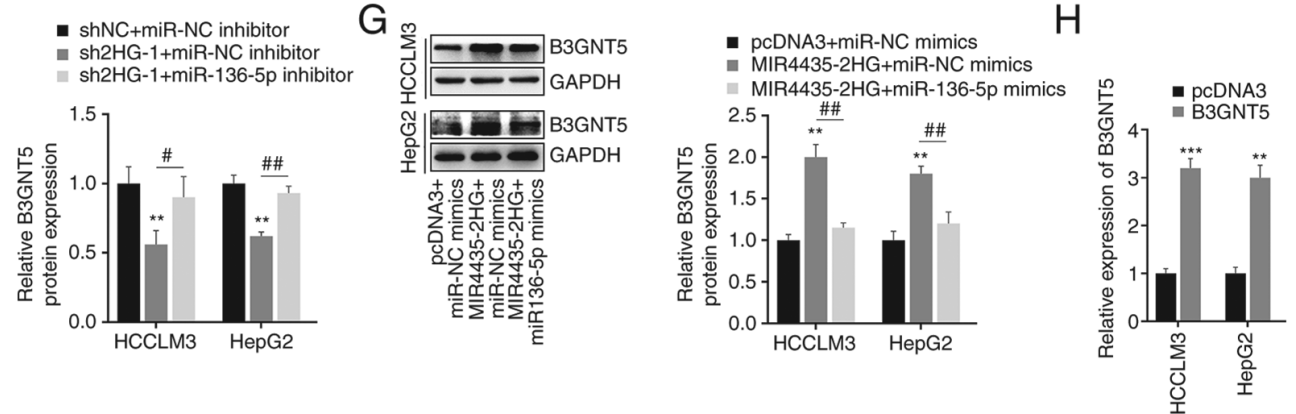

$\rightarrow$ shNC+pcDNA3 $\because$ sh2HG-1+pcDNA3

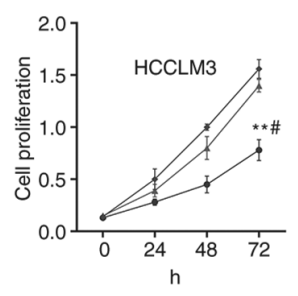

$\rightarrow$ shNC+pcDNA3 $\rightarrow$ sh2HG-1+pcDNA3
- sh2HG-1+B3GNT5

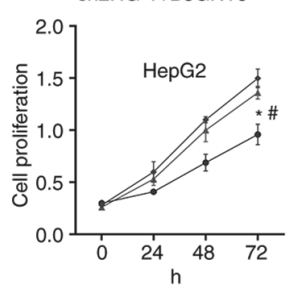

K

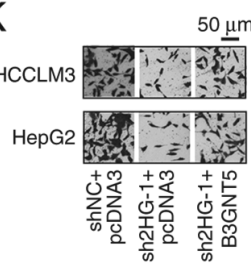

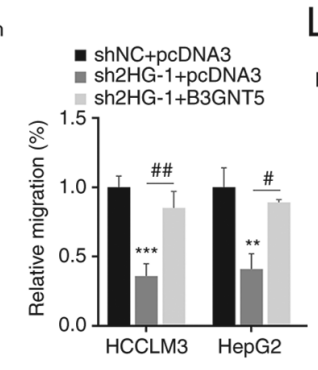

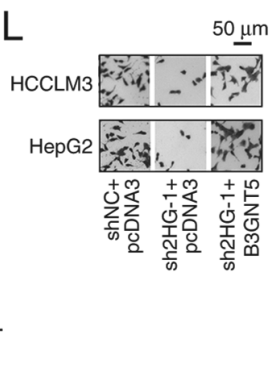

J
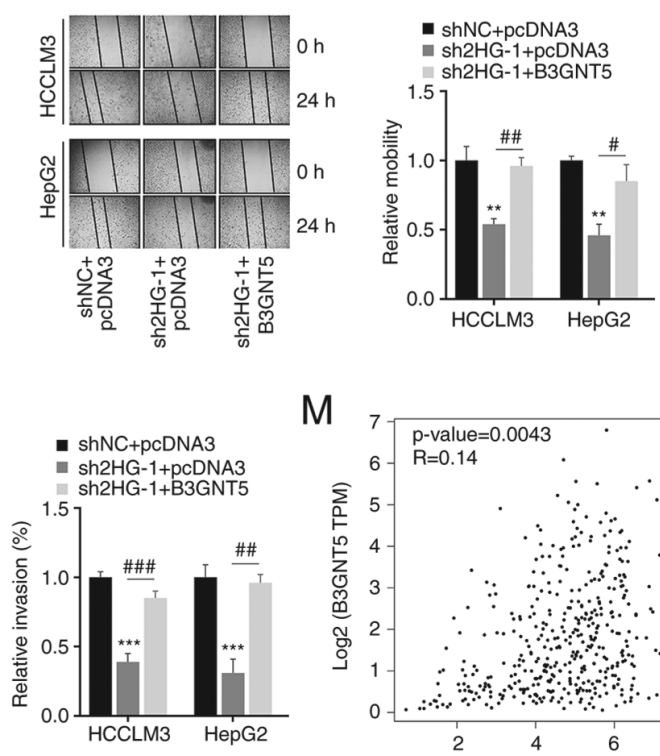

M

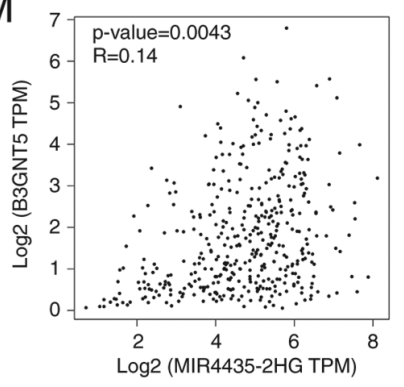

Figure 5. MIR4435-2HG promotes B3GNT5 expression in liver cancer cells. (A) Dual-luciferase reporter assay was used to detect the binding between B3GNT5 and miR-136-5p. ${ }^{* * *} \mathrm{P}<0.001$ vs. miRNA NC. (B and C) mRNA and protein expression levels of B3GNT5 in liver cancer and adjacent normal tissues. ${ }^{* *} \mathrm{P}<0.01$ vs. normal tissues. (D and E) mRNA and protein expression levels of B3GNT5 in normal liver cell line and liver cancer cell lines. ${ }^{~} \mathrm{P}<0.05$, ${ }^{* *} \mathrm{P}<0.01$ vs. THLE-2 cells. (F) MIR4435-2HG regulated B3GNT5 protein expression by targeting miR-136-5p, as shown following transfection with sh2HG-1 and miR-136-5p inhibitor. ${ }^{* *} \mathrm{P}<0.01$ vs. shNC + miR-NC inhibitor; ${ }^{*} \mathrm{P}<0.05,{ }^{\# \#} \mathrm{P}<0.01$ vs. sh2HG + miR-NC inhibitor. (G) MIR4435-2HG regulated B3GNT5 protein expression by targeting miR-136-5p, as shown following transfection with MIR4435-2HG and miR-136-5p mimics. ${ }^{* *} \mathrm{P}<0.01$ vs. pcDNA3 + miR-NC mimics; ${ }^{\# \#} \mathrm{P}<0.01$ vs. MIR4435-2HG + miR-NC mimics. (H) Overexpression efficiency of B3GNT5. ${ }^{* *} \mathrm{P}<0.01,{ }^{* * * *} \mathrm{P}<0.001$ vs. pcDNA3. (I-L) Knockdown of MIR4435-2HG significantly suppressed the proliferation, migration and invasion of liver cancer cells, these effects were reversed by the overexpression of B3GNT5. Magnification, $\mathrm{x} 50 .{ }^{*} \mathrm{P}<0.05,{ }^{* *} \mathrm{P}<0.01,{ }^{* * *} \mathrm{P}<0.001$ vs. shNC + pcDNA3, ${ }^{*} \mathrm{P}<0.05,{ }^{\# \#} \mathrm{P}<0.01,{ }^{\# \# \#} \mathrm{P}<0.001$ vs. sh2HG-1 + pcDNA3. (M) Data in the Gene Expression Profiling Interactive Analysis database demonstrated the positive relationship between B3GNT5 and MIR4435-2HG in liver cancer. miR/miRNA, microRNA; WT, wild-type; MUT, mutant; NC, negative control; sh, short hairpin RNA; sh2HG, shMIR4435-2HG.

for heatmap analysis of expression using the UALCAN database (data not shown). It was found that seven genes (PBX3, B3GNT5, FZD4, DRAM1, PTPDC1, SFXN3, MLLT3) had the highest expression in liver cancer; thus, the seven genes were selected for further analysis. Next, the higher expression levels of seven target genes were found in liver cancer tissues compared with those in normal tissues from patients with liver cancer using the UALCAN database (Fig. 4B), which indicated that the higher expression levels of the seven target genes may be related to liver cancer. To the best of our knowledge, the function of B3GNT5 has not yet been reported in liver cancer; therefore, the present study focused on B3GNT5 and explored its function in liver cancer. First, the expression of B3GNT5 was determined using the GEPIA database and it was revealed to be highly expressed in liver cancer tissues compared with in adjacent normal tissues (Fig. 4C). Patients with higher B3GNT5 expression levels had shorter OS and DFS (Fig. 4D). Furthermore, the SNV of six of the 
seven targets was determined; notably, the SNV of DRAM1 is not currently included in the GSCA database. The results revealed that four of the six genes (PTPDC1, MLLT3, FZD4 and B3GNT5) exhibited SNV in liver cancer (Fig. 4E). A missense mutation of B3GNT5 was observed in liver cancer (Fig. 4F). In addition, CNV was analyzed; the results showed that B3GNT5 also exhibited CNV in liver cancer (Fig. 4G). In most cases, the cancer progression is accompanied by changes in gene methylation levels (21-24). By analyzing the GSCA database, the B3GNT5 methylation levels across TCGA cancer types are presented in Fig. 4H. Methylation levels of the five of the seven targets were decreased and negatively associated with mRNA expression; methylation differences of FZD4 and SFXN3 in LIHC are not currently included in the GSCA database (Fig. 4I and J). Our previous results revealed that the expression levels of these target genes were elevated in liver cancer, and the decrease in methylation levels may be conducive to the increase in their expression levels. These findings indicated that these target genes may be mutated in liver cancer and their expression levels may also be increased in liver cancer. Furthermore, Spearman's correlation analysis indicated a negative correlation between B3GNT5 methylation and expression at the mRNA level (Fig. 4K), which may indicate that the decrease in B3GNT5 methylation contributes to the expression of B3GNT5 in liver cancer. These results showed that B3GNT5 expression was increased in liver cancer. Therefore, B3GNT5 may act as an oncogene in liver cancer.

MIR4435-2HG promotes B3GNT5 expression by regulating $m i R-136-5 p$. In general, miRNAs exercise their role by targeting mRNA and inhibiting protein activity $(25,26)$. Thus, the association between B3GNT5 and miR-136-5p was confirmed using a dual-luciferase reporter assay (Fig. 5A). The mRNA and protein expression levels of B3GNT5 were detected using RT-qPCR and western blotting, respectively. It was found that B3GNT5 expression was abnormally increased in liver cancer tissues and cells (Figs. 5B-E and S1C and D). Furthermore, western blot analysis suggested that MIR4435-2HG positively regulated B3GNT5 expression by inhibiting miR-136-5p in liver cancer cells (Fig. 5F and G). To further verify that MIR4435-2HG promotes liver cancer progression by mediating miR-136-5p and B3GNT5 expression, cancer cells were transfected with sh2HG-1 and B3GNT5. B3GNT5 overexpression was confirmed, as presented in Fig. 5H. It was found that MIR4435-2HG knockdown significantly inhibited cell proliferation; however, the overexpression of B3GNT5 partially rescued the effect of MIR4435-2HG knockdown (Fig. 5I). The decreased cell migration and invasion abilities caused by sh2HG-1 were also reversed by the overexpression of B3GNT5 (Fig. 5J-L). Consistent with the aforementioned results, it was found that the expression of MIR4435-2HG was slightly positively correlated with that of B3GNT5 in liver cancer specimens (Fig. 5M). Thus, these results suggested a critical role of the MIR4435-2HG/miR-136-5p/B3GNT5 axis in liver cancer.

\section{Discussion}

Increasing evidence has indicated that abnormally expressed lncRNAs play key roles in various types of cancer (27). In the present study, the expression levels of MIR4435-2HG were evaluated using TCGA database and RT-qPCR assays. It was found that the expression levels of MIR4435-2HG were significantly upregulated in liver cancer tissues and cell lines, which was consistent with previous reports $(14,15,28)$. Further analysis indicated that MIR4435-2HG overexpression was associated with cancer metastasis and poor prognosis in patients with liver cancer. Additionally, to explore the function of MIR4435-2HG in liver cancer progression, silencing or overexpression of MIR4435-2HG in liver cancer cells was performed. It was observed that silencing MIR4435-2HG reduced the proliferation, migration and invasion of liver cancer cells, whereas its overexpression resulted in the opposite effect on the malignant phenotype of liver cancer cells. Regarding the mechanism of action, the results showed that MIR4435-2HG promoted liver cancer tumorigenesis by binding miR-136-5p to activate the expression of B3GNT5. In previous studies, MIR4435-2HG expression has been found to be elevated in cancer tissues compared with in adjacent normal tissues in patients with liver cancer, and its overexpression has been reported to promote cell proliferation by upregulating miRNA-487a expression (15). In addition, MIR4435-2HG has been observed to be highly expressed in liver cancer tissues, and the malignant progression of liver cancer may be promoted by the MIR4435-2HG/miR-22-3p/YWHAZ axis (14).

It is commonly known that lncRNAs partially mediate cancer progression by acting as ceRNAs. For example, knockdown of IncRNA SOX2OT has been reported to induce cell apoptosis, and promote the proliferation, migration and invasion of glioblastoma stem cells via the SOX2OT-miR-194-5p/miR-122-SOX3-TDGF-1 signaling pathway (29). Furthermore, the increased expression of IncRNA RP11-436H11.5 affected cell proliferation and invasion in vitro and in vivo by regulating miR-335-5p expression in renal cell carcinoma (30). In addition, HOXD-AS1 has been demonstrated to facilitate cancer metastasis and inhibit apoptosis via the miR19a/ARHGAP11A signaling axis in HCC (31). In the present study, the potential target miRNAs of MIR4435-2HG were predicted using bioinformatics tools. It has been revealed that miR-136-5p expression may be markedly decreased in various types of cancer. For example, miR-136-5p may inhibit HCC cell proliferation, migration and invasion by targeting the WNK1 pathway (32). It may also inhibit lung squamous cell carcinoma progression by deactivating ROCK1 (33). Furthermore, miR-136-5p has been reported to regulate the expression of IRX5 to suppress cell proliferation in HCC (34). The present study reported that MIR4435-2HG could function as a ceRNA of miR-136-5p. The mutual negative regulatory association between MIR4435-2HG and miR-136-5p was elucidated. Furthermore, it was demonstrated that the MIR4435-2HG knockdown-mediated inhibition of cell proliferation, migration and invasion was reversed by miR-136-5p silencing. Bioinformatics tools were used in the current study to search for candidate targets of miR-136-5p, and B3GNT5 was used for expression analysis. To the best of our knowledge, the function of B3GNT5 in liver cancer development has not been previously reported. In the present study, mutations in B3GNT5 in liver cancer were identified through online database analysis. The expression of B3GNT5 in liver cancer was observed to be 
upregulated, but its methylation level was decreased, indicating that its increased expression in liver cancer may be beneficial for cancer progression. Therefore, the investigation of B3GNT5 methylation and its role in liver cancer may be a potential future research direction. From the perspective of gene expression regulation, the epigenetic results indeed found that the expression of B3GNT5 was altered in liver cancer, which indicated that B3GNT5 may play an important role in the occurrence and development of liver cancer and is worthy of further study. Therefore, B3GNT5 may be a candidate target for the treatment of liver cancer. Additionally, it was found that B3GNT5 was highly expressed in liver cancer tissues and cell lines, and was related to poor prognosis of patients. Moreover, B3GNT5 expression was regulated by the MIR4435-2HG/miR-136-5p axis. Functionally, the overexpression of B3GNT5 relieved the sh-2HG-1-induced inhibition of cell proliferation, migration and invasion. These results were consistent with the previous study in human glioma in which B3GNT5 was revealed to act as a tumor promoter (35). However, a major limitation of the current study is that B3GNT5-related signaling pathways and the effect of MIR4435-2HG in liver cancer in vivo were not comprehensively studied and thus will be explored further in the future.

In summary, it was found that MIR4435-2HG was highly expressed in liver cancer tissues and cells. These data indicated the oncogenic role of MIR4435-2HG in liver cancer by competitively sponging miR-136-5p to mediate B3GNT5 expression. To the best of our knowledge, this was the first study to reveal the potential mechanism of the MIR4435-2HG/miR-136-5p/B3GNT5 axis in liver cancer. The present study may provide a potential biomarker for the diagnosis and treatment of liver cancer.

\section{Acknowledgements}

Not applicable.

\section{Funding}

No funding was received.

\section{Availability of data and materials}

The datasets used and/or analyzed during the current study are available from the corresponding author on reasonable request.

\section{Authors' contributions}

GXi, YZ and BL designed and conceived the study. YZ, BL, $\mathrm{GXu}$ and $\mathrm{CH}$ conducted the experiments. All authors analyzed the data. GXi, YZ and BL drafted the manuscript. All authors have read and approved the final manuscript. GXi and YZ confirmed the authenticity of all the raw data.

\section{Ethics approval and consent to participate}

This study was approved by the Ethics Committee of the Second Hospital of Tianjin Medical University (approval no. KY2020K093; Tianjin, China). All patients provided written informed consent prior to the study.

\section{Patient consent for publication}

Not applicable.

\section{Competing interests}

The authors declare that they have no competing interests.

\section{References}

1. Satriano L, Lewinska M, Rodrigues PM, Banales JM and Andersen JB: Metabolic rearrangements in primary liver cancers: Cause and consequences. Nat Rev Gastroenterol Hepatol 16: 748-766, 2019.

2. Anwanwan D, Singh SK, Singh S, Saikam V and Singh R: Challenges in liver cancer and possible treatment approaches. Biochim Biophys Acta Rev Cancer 1873: 188314, 2020.

3. Harris PS, Hansen RM, Gray ME, Massoud OI, McGuire BM and Shoreibah MG: Hepatocellular carcinoma surveillance: An evidence-based approach. World J Gastroenterol 25: 1550-1559, 2019.

4. Aldrighetti L, Pulitano C, Catena M, Arru M, Guzzetti E, Halliday J and Ferla G: Liver resection with portal vein thrombectomy for hepatocellular carcinoma with vascular invasion. Ann Surg Oncol 16: 1254, 2009.

5. Li J, Li Z, Zheng W, Li X, Wang Z, Cui Y and Jiang X: LncRNA-ATB: An indispensable cancer-related long noncoding RNA. Cell Prolif 50: e12381, 2017.

6. Ulitsky I and Bartel DP: lincRNAs: Genomics, evolution, and mechanisms. Cell 154: 26-46, 2013.

7. Shi X, Sun M, Liu H, Yao Y and Song Y: Long non-coding RNAs: A new frontier in the study of human diseases. Cancer Lett 339: 159-166, 2013

8. Chen C, Zheng H, Luo Y, Kong Y, An M, Li Y, He W, Gao B, Zhao Y, Huang $\mathrm{H}$, et al: SUMOylation promotes extracellular vesicle-mediated transmission of lncRNA ELNAT1 and lymph node metastasis in bladder cancer. J Clin Invest 131: e146431, 2021.

9. Qin Y, Hou Y, Liu S, Zhu P, Wan X, Zhao M, Peng M, Zeng H, Li Q, Jin T, et al: A novel long non-coding RNA lnc030 maintains breast cancer stem cell stemness by stabilizing SQLE mRNA and increasing cholesterol synthesis. Adv Sci (Weinh) 8: 2002232, 2020.

10. Zhang F, Wang H, Yu J, Yao X, Yang S, Li W, Xu L and Zhao L: LncRNA CRNDE attenuates chemoresistance in gastric cancer via SRSF6-regulated alternative splicing of PICALM. Mol Cancer 20: 6, 2021.

11. Dong X, Yang Z, Yang H, Li D and Qiu X: Long non-coding RNA MIR4435-2HG promotes colorectal cancer proliferation and metastasis through miR-206/YAP1 axis. Front Oncol 10: 160, 2020.

12. Wang H, Wu M, Lu Y, He K, Cai X, Yu X, Lu J and Teng L: LncRNA MIR4435-2HG targets desmoplakin and promotes growth and metastasis of gastric cancer by activating Wnt $/ \beta$-catenin signaling. Aging (Albany NY) 11: 6657-6673, 2019.

13. Yang M, He X, Huang X, Wang J, He Y and Wei L: LncRNA MIR4435-2HG-mediated upregulation of TGF- $\beta 1$ promotes migration and proliferation of nonsmall cell lung cancer cells. Environ Toxicol 35: 582-590, 2020.

14. Shen X, Ding Y, Lu F, Yuan H and Luan W: Long noncoding RNA MIR4435-2HG promotes hepatocellular carcinoma proliferation and metastasis through the miR-22-3p/YWHAZ axis. Am J Transl Res 12: 6381-6394, 2020.

15. Kong Q, Liang C, Jin Y, Pan Y, Tong D, Kong Q and Zhou J: The lncRNA MIR4435-2HG is upregulated in hepatocellular carcinoma and promotes cancer cell proliferation by upregulating miRNA-487a. Cell Mol Biol Lett 24: 26, 2019.

16. Livak KJ and Schmittgen TD: Analysis of relative gene expression data using real-time quantitative PCR and the 2(-Delta Delta C(T)) method. Methods 25: 402-408, 2001.

17. Wang S, Zhang X, Li Z, Wang W, Li B, Huang X, Sun G, Xu J, Li Q, Xu Z, et al: Circular RNA profile identifies circOSBPL10 as an oncogenic factor and prognostic marker in gastric cancer. Oncogene 38: 6985-7001, 2019.

18. Han C, Fu Y, Zeng N, Yin J and Li Q: LncRNA FAM83H-AS1 promotes triple-negative breast cancer progression by regulating the miR-136-5p/metadherin axis. Aging (Albany NY) 12: 3594-3616, 2020 
19. Kang W, Wang Q, Dai Y, Wang H, Wang M, Wang J, Zhang D, Sun P, Qi T, Jin X and Cui Z: Hypomethylation of PlncRNA-1 promoter enhances bladder cancer progression through the miR-136-5p/Smad3 axis. Cell Death Dis 11: 1038, 2020.

20. Wang Z, Huang C, Zhang A, Lu C and Liu L: Overexpression of circRNA_100290 promotes the progression of laryngeal squamous cell carcinoma through the miR-136-5p/RAP2C axis. Biomed Pharmacother 125: 109874, 2020.

21. Xu N, Wu YP, Ke ZB, Liang YC, Cai H, Su WT, Tao X, Chen SH, Zheng QS, Wei Y and Xue XY: Identification of key DNA methylation-driven genes in prostate adenocarcinoma: An integrative analysis of TCGA methylation data. J Transl Med 17: $311,2019$.

22. Kresovich JK, Joyce BT, Gao T, Zheng Y, Zhang Z, Achenbach CJ, Murphy RL, Just AC, Shen J, Yang H, et al: Promoter methylation of PGC1A and PGC1B predicts cancer incidence in a veteran cohort. Epigenomics 10: 733-743, 2018.

23. Weisenberger DJ, Liang G and Lenz HJ: DNA methylation aberrancies delineate clinically distinct subsets of colorectal cancer and provide novel targets for epigenetic therapies. Oncogene 37 566-577, 2018.

24. Kwok CT, Marshall AD, Rasko JE and Wong JJ: Genetic alterations of $\mathrm{m}^{6} \mathrm{~A}$ regulators predict poorer survival in acute myeloid leukemia. J Hematol Oncol 10: 39, 2017.

25. Fernandes T, Barretti DL, Phillips MI and Menezes Oliveira E: Exercise training prevents obesity-associated disorders: Role of miRNA-208a and MED13. Mol Cell Endocrinol 476: 148-154, 2018.

26. Correia de Sousa M, Gjorgjieva M, Dolicka D, Sobolewski C and Foti M: Deciphering miRNAs' action through miRNA editing. Int J Mol Sci 20: 6249, 2019

27. Klingenberg M, Matsuda A,Diederichs $S$ and Patel T: Non-coding RNA in hepatocellular carcinoma: Mechanisms, biomarkers and therapeutic targets. J Hepatol 67: 603-618, 2017.

28. Wang B, Tang D, Zhang $\mathrm{Z}$ and Wang Z: Identification of aberrantly expressed lncRNA and the associated TF-mRNA network in hepatocellular carcinoma. J Cell Biochem 121: 1491-1503, 2020.
29. Su R, Cao S, Ma J, Liu Y, Liu X, Zheng J, Chen J, Liu L, Cai H, Li Z, et al: Knockdown of SOX2OT inhibits the malignant biological behaviors of glioblastoma stem cells via up-regulating the expression of miR-194-5p and miR-122. Mol Cancer 16: 171, 2017.

30. Wang K, Jin W, Song Y and Fei X: LncRNA RP11-436H11.5, functioning as a competitive endogenous RNA, upregulates BCL-W expression by sponging miR-335-5p and promotes proliferation and invasion in renal cell carcinoma. Mol Cancer 16: 166, 2017.

31. Lu S, Zhou J, Sun Y, Li N, Miao M, Jiao B and Chen H: The noncoding RNA HOXD-AS1 is a critical regulator of the metastasis and apoptosis phenotype in human hepatocellular carcinoma. Mol Cancer 16: 125, 2017.

32. Dong H, Jian P, Yu M and Wang L: Silencing of long noncoding RNA LEF1-AS1 prevents the progression of hepatocellular carcinoma via the crosstalk with microRNA-136-5p/WNK1. J Cell Physiol 235: 6548-6562, 2020.

33. Zhang W, Shi J, Cheng C and Wang H: CircTIMELESS regulates the proliferation and invasion of lung squamous cell carcinoma cells via the miR-136-5p/ROCK1 axis. J Cell Physiol 235: 5962-5971, 2020.

34. Zhu L, Liu Y, Chen Q, Yu G, Chen J, Chen K, Yang N, Zeng T, Yan S, Huang A and Tang H: Long-noncoding RNA colorectal neoplasia differentially expressed gene as a potential target to upregulate the expression of IRX 5 by miR-136-5P to promote oncogenic properties in hepatocellular carcinoma. Cell Physiol Biochem 50: 2229-2248, 2018.

35. Jeong HY, Park SY, Kim HJ, Moon S, Lee S, Lee SH and Kim SH: B3GNT5 is a novel marker correlated with stem-like phenotype and poor clinical outcome in human gliomas. CNS Neurosci Ther 26: 1147-1154, 2020.

This work is licensed under a Creative Commons Attribution-NonCommercial-NoDerivatives 4.0 International (CC BY-NC-ND 4.0) License. 